\title{
The Cardiac R-R Variation and Sympathetic Skin Response in the Intensive Care Unit
}

\author{
Charles Bolton, Jillian Thompson, Linda Bernardi, Christopher Voll, \\ Bryan Young
}

\begin{abstract}
Background and Purpose: The central and peripheral nervous systems are often affected in intensive care unit (ICU) patients, especially those with prolonged assisted ventilation and sepsis or systemic inflammatory response syndrome (SIRS). The autonomic nervous system, however, has been under-investigated in such patients. We evaluated autonomic nervous system (ANS) function in 29 ICU patients with various neurological disorders. Methods: Testing involved cardiac R-R variation (CRRV) as an index of parasympathetic function and the sympathetic skin response (SSR) for sympathetic assessment. Results: Only those 8 patients with sepsis-related neuropathy or encephalopathy had abnormal CRRV, while the SSR was absent in all but 2 patients. Conclusions: Our preliminary study revealed a high incidence of autonomic dysfunction in ICU patients with various neurological disorders.
\end{abstract}

RÉSUMÉ: La variation cardiaque R-R et la réponse cutanée sympathique chez des patients de l'unité de soins intensifs. Contexte et objectif : Le système nerveux central et le système nerveux périphérique sont souvent atteints chez les patients hospitalisés à l'unité de soins intensifs (USI), surtout chez ceux qui sont sous ventilation assistée prolongée et ceux qui sont atteints de septicémie ou d'un syndrome de réaction inflammatoire généralisée (SIRS). Cependant, le système nerveux autonome (SNA) a été peu étudié chez ces patients. Nous avons étudié la fonction du SNA chez 29 patients de l'USI atteints de différents problèmes neurologiques. Méthodes : Nous avons étudié la variation de l'intervalle R-R (VIRR) comme indice de la fonction parasympathique et la réponse cutanée sympathique (RCS) pour la fonction sympathique. Résultats : Seuls les 8 patients atteints de neuropathie ou d'encéphalopathie reliée à une septicémie avaient une VIRR anormale et la RCS était présente chez deux patients seulement. Conclusions : Cette étude préliminaire a révélé une incidence élevée de dysfonction autonome chez les patients atteints de différents troubles neurologiques qui sont hospitalisés à l’USI.

Can. J. Neurol. Sci. 2007; 34: 313-315

Clinical and electrophysiological studies reveal a high incidence of nervous system dysfunction in patients treated in major medical and surgical intensive care units (ICU's). ${ }^{1,2}$ This dysfunction is especially common in patients who suffer from a systemic inflammatory response syndrome (SIRS) as a critical illness. ${ }^{3}$ Systemic inflammatory response syndrome occurs in at least $50 \%$ of patients who are treated on ventilators in the ICU for more than one week. ${ }^{4}$ Septic encephalopathy and critical illness polyneuropathy (CIP) both occur in $70 \%$ of SIRS patients. ${ }^{5}$ Critical illness myopathy is especially prominent in patients who, in addition to suffering from SIRS, receive neuromuscular blocking agents and steroids. ${ }^{6}$ The amplitude of the QRS complex in the electrocardiogram is reduced in critically ill patients. ${ }^{7}$
Since SIRS has widespread central and peripheral nervous system effects, we decided to examine the autonomic nervous system prospectively. The parasympathetic nervous system can be assessed by measuring the cardiac R-R variation (CRRV) and the sympathetic pathways are evaluated by using the

From the Division of Neurology, University of Western Ontario (CB, JT, LB, BY), London, Ontario; The University of Saskatchewan (CV), Saskatoon, Saskatchewan, Canada.

Received November 6, 2006. AcCePted in finAl Form March 19, 2007. Reprint requests to: G.B. Young, Department of Clinical Neurological Sciences, University Hospital, 339 Windermere Road, London, Ontario, N6A 5A5, Canada. 
sympathetic skin response (SSR) that mediates cutaneous cholinergic (sudomotor) function via both central and peripheral pathways. ${ }^{8-10}$ The CRRV and SSR have been shown to be abnormal in patients outside the ICU who have a wide variety of disorders affecting these pathways. ${ }^{9,11}$

\section{Methods}

Studies were performed on patients in a 30 bed medicalsurgical ICU. Neurological and electrophysiological consultations were requested to investigate a variety of possible central and peripheral nervous system (CNS and PNS) disorders according to previously documented techniques. ${ }^{12}$ Limb temperature was monitored routinely. For CNS disorders, somatosensory evoked potentials were utilized. For PNS disorders motor and sensory nerve conduction and the needle electrode myography were used. There were 18 men and 15 women ranging in age from 19 to 83 years of age; 11 patients were over 60 years of age. The predominant neurological diagnoses were septic encephalopathy (2), anoxic- ischemic encephalopathy (1), Parkinson disease (1), drug induced extrapyramidal syndrome (1), traumatic encephalopathy (3), traumatic quadriplegia (2), critical illness polyneuropathy (14), and myopathy (5). At the time of testing patients were not receiving adrenergic blockers or drugs with anticholinergic properties that might affect the CRRV and SSR. Critically ill patients had widespread organ dysfunction, glucose intolerance and other manifestations of SIRS. They had a variety of reasons for ICU admission.

Both the CRRV and the SSR were measured according to the technique of Shahani et al, ${ }^{10}$ and were performed on an
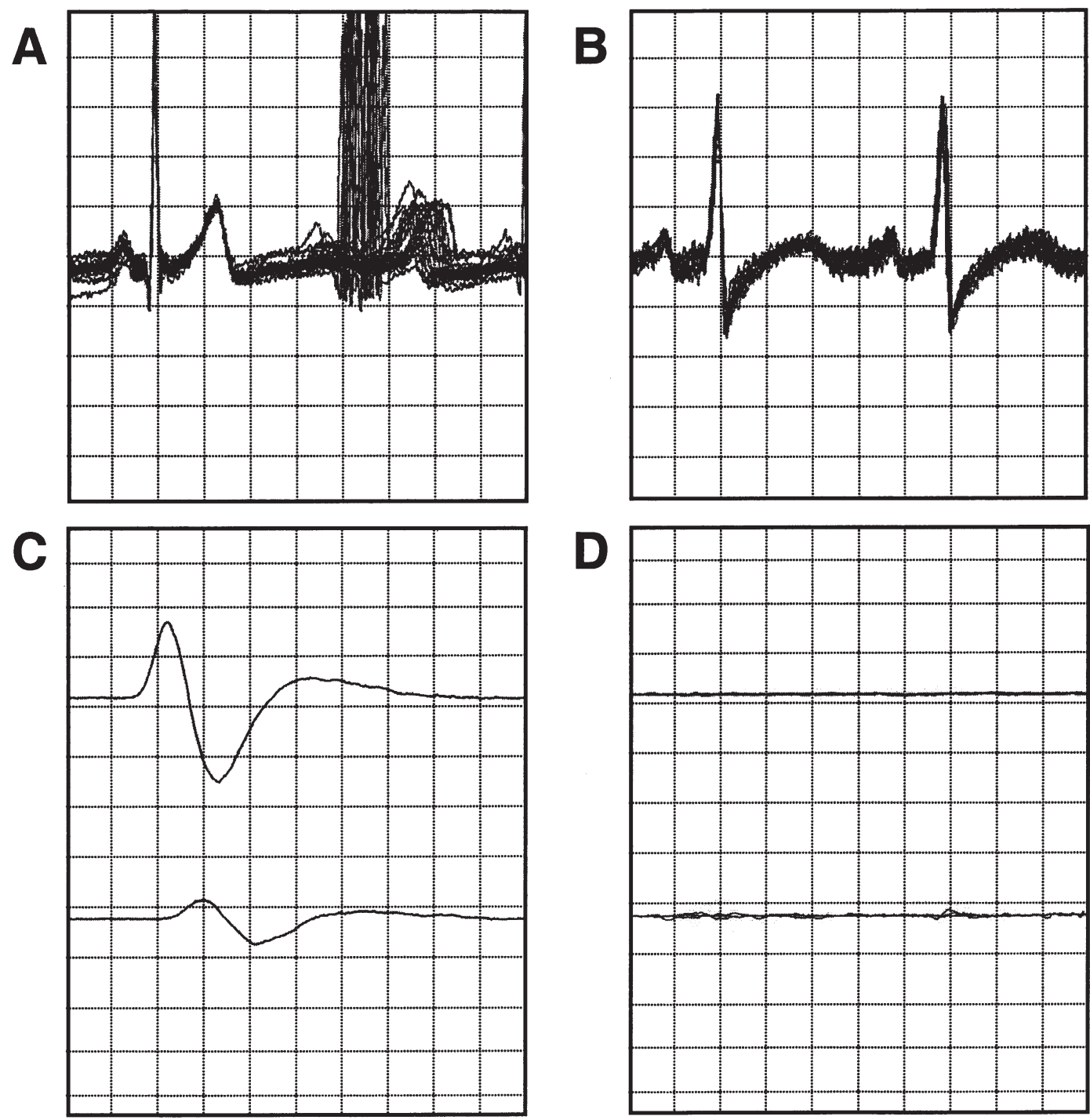

Figure: The normal result in healthy persons is shown in A for the CRRV and C for the SSR (upper trace is for the hand and the lower trace is the foot.) The abnormal response for the CRRV is shown in B (a fixed R-R interval) and for SSR in D (absent response from the hand and foot.) (Calibration A$200 \mathrm{~ms}$ and $200 \mu \mathrm{V} /$ div; $B-200 \mathrm{~ms}$ and $100 \mu \mathrm{V} /$ div; $C-1.0 \mathrm{sec}$ and $500 \mu \mathrm{V} / \mathrm{div} ; \mathrm{D}-1.0 \mathrm{sec}$ and $200 \mu \mathrm{V} / \mathrm{div}$.) 
Advantage EMG system (Advantage Medical, London, Ontario, Canada) with special software designed for these procedures. Each patient was on a ventilator and testing was done while the ventilator was operating. The SSR was tested before any other stimulus was given to the patient to avoid habituation. The stimulus was applied to the left median nerve at the wrist randomly with duration of 0.2 milliseconds and 15-50 milliamps intensity. Five sets of stimuli were given at greater than 30 second intervals. Responses were recorded simultaneously with surface electrodes over the contralateral palm and sole of the foot. Sympathetic skin response was performed at filter settings between 0.5 and $100 \mathrm{~Hz}$ and gain between 200 and $500 \mu \mathrm{V} /$ div. Sympathetic skin response was considered abnormal only if it was entirely absent at both the hand and the foot. Cardiac R-R variation was then tested, with filter settings between 0.5 and 100 $\mathrm{Hz}$ and gain from 100-500 $\mu \mathrm{V} / \mathrm{div}$, and expressed as a percentage of the average RR interval and was considered abnormal if the variation was less than either $12 \%$ or less than $5 \%$ for patients over 60 years of age.

Two patients, who had been studied while on ventilators in the ICU and were found to have absent SSR's, were tested one month after discharge from the ICU while off the ventilator.

\section{Results}

Eight of the 29 patients had an abnormal CRRV (Figure). Six of these had CIP (mild in 5 and moderate in 1) and 2 had mild septic encephalopathy. ${ }^{1}$ The SSR was absent (Figure) in all patients (see list in methods) except for a 73 year old female with anoxic-ischemic encephalopathy, and a 54 year old female who was on the ventilator briefly for a drug-induced extrapyramidal syndrome.

The median nerve sensory action potential (SNAP) recorded at the index finger from wrist stimulation was normal in 21, reduced in 1 , absent in 1 and, for several technical reasons, not tested in 6 patients.

Two patients with moderate/severe CIP had absent SSRs while on the ventilator in the ICU and when tested one month later, while off the ventilator.

The typical results of the CRRV and the SSR for healthy persons and patients are shown in the Figure.

\section{DiscuSSION}

The study revealed a high incidence of dysfunction in critically ill patients involving both the parasympathetic (measured by the CRRV) and sympathetic (measured by the SSR) pathways, in the central and peripheral nervous systems. It is known that sepsis SIRS causes widespread dysfunction of the brain, peripheral nerve and muscle in the form of septic encephalopathy, critical illness polyneuropathy and myopathy. ${ }^{13}$ It has been shown that cardiac muscle is also involved manifested as reduction in the amplitude of the QRS complex of the electrocardiogram. ${ }^{7}$ Examination of the sympathetic chain at autopsy in critically ill patients reveals axonal degeneration. ${ }^{12}$ However, studies have not been previously performed on the parasympathetic connections to the heart and it has not been previously determined whether unmyelinated fibres of the peripheral nervous system are involved. There is dysfunction of the parasympathetic and sympathetic systems in critically ill patients, which may relate to septic encephalopathy and CIP.
The absent SSR may explain the tendency to hypotension in the critically ill patients. ${ }^{9}$ Dysfunction of sympathetic pathways in the CNS probably explains the absent SSR in patients with anoxic-ischemic encephalopathy, traumatic encephalopathy, traumatic quadriplegia and Parkinson's disease who did not have SIRS.

It seems unlikely that habituation from the repetitive action of the ventilator stimulating the lungs would cause the absent SSR since two of our patients with moderate to severe critical illness polyneuropathy continued to have an absent SSR one month after discharge from the ICU and off the ventilator. The median nerve SNAP was normal in 21 patients, making it unlikely that dysfunction of the afferent limb of the SSR accounted for the absent SSR. Furthermore, none were on adrenergic blockers or drugs with anticholinergic properties that would block or blunt the SSR. This was a preliminary study; prospective studies should include repeat testing during and after the ICU stay in well defined patient groups. Recording of the quantitative sudomotor axon reflex ${ }^{8}$ would further establish the presence of sympathetic dysfunction and determine if it is proximal or distal to the sympathetic ganglia.

\section{REFERENCES}

1. Bolton CF, Young GB. The neurological consultation and neurological syndromes. In: Bolton $\mathrm{CF}$, Young GB, editors. Balliere's clinical neurology: international practice and research. Critical Care. London:Balliere Tindall; 1996. p. 447-76.

2. Bolton CF. Neuromuscular manifestations of critical illness. Muscle Nerve. 2005;32:140-63.

3. Bolton CF. Sepsis and the systemic inflammatory response syndrome: neuromuscular manifestations. Crit Care Med. 1996; 24:1408-16.

4. Tran DD, Groeneveld, AB, van der Meulen, J, Nauta JJ, Strack van Schijndel RJ, Thijs LG. Age, chronic disease, sepsis, organ system failure and mortality in a medical intensive care unit. Crit Care Med. 1990;18:474-9.

5. Bolton CF, Young GB, Zochodone DW. The neurological complications of sepsis. Ann Neurol. 1993;33:94-100.

6. Lacomis D, Zochodne DW, Bird SJ. Critical illness myopathy. Muscle Nerve. 2000;23:1785-8.

7. Rich MM, McGarvey ML, Teener JW, Frame LH. ECG changes during septic shock. Cardiology. 2002;97:187-96.

8. Low PA, Caskey PA, Zuck RR, Fealey RD, Dyct PJ. Quantitative sudomotor axon reflex test in normal and neuropathic subjects. Ann Neurol. 1983;14:573-80.

9. Ravitz JM, AAEM Minimonograph \#48. Autonomic nervous system testing. Muscle Nerve. 1997;20:919-37.

10. Shahani BT, Day TJ, Cros D, Kahlil N, Kneebone CS. R-R Interval variation and the sympathetic skin response in the assessment of autonomic function in peripheral neuropathy. Arch Neurol. 1990; 47:659-64.

11. Kneebone CS. R-R interval variation and the sympathetic skin response in the assessment of autonomic function in peripheral neuropathy. Arch Neurol. 1990;47:659-64.

12. Zochodne DW, Bolton CF, Wells GA, Gilbert JJ, Hahn AF, Brown JD, et al. Critical illness polyneuropathy. A complication of sepsis and multiple organ failure. Brain. 1987;110:819-41.

13. Bolton CF. Critical illness polyneuropathy, In: Noseworthy JH, editor. Neurological therapeutics. London: Martin Dunitz; 2003. p. 2229-36. 